\title{
Foreign body granulomas after a craniotomy for meningioma
}

\author{
Kessarin Panichpisal MD, Guy Aristide MD, Craig Linden MD, John Miller MD
}
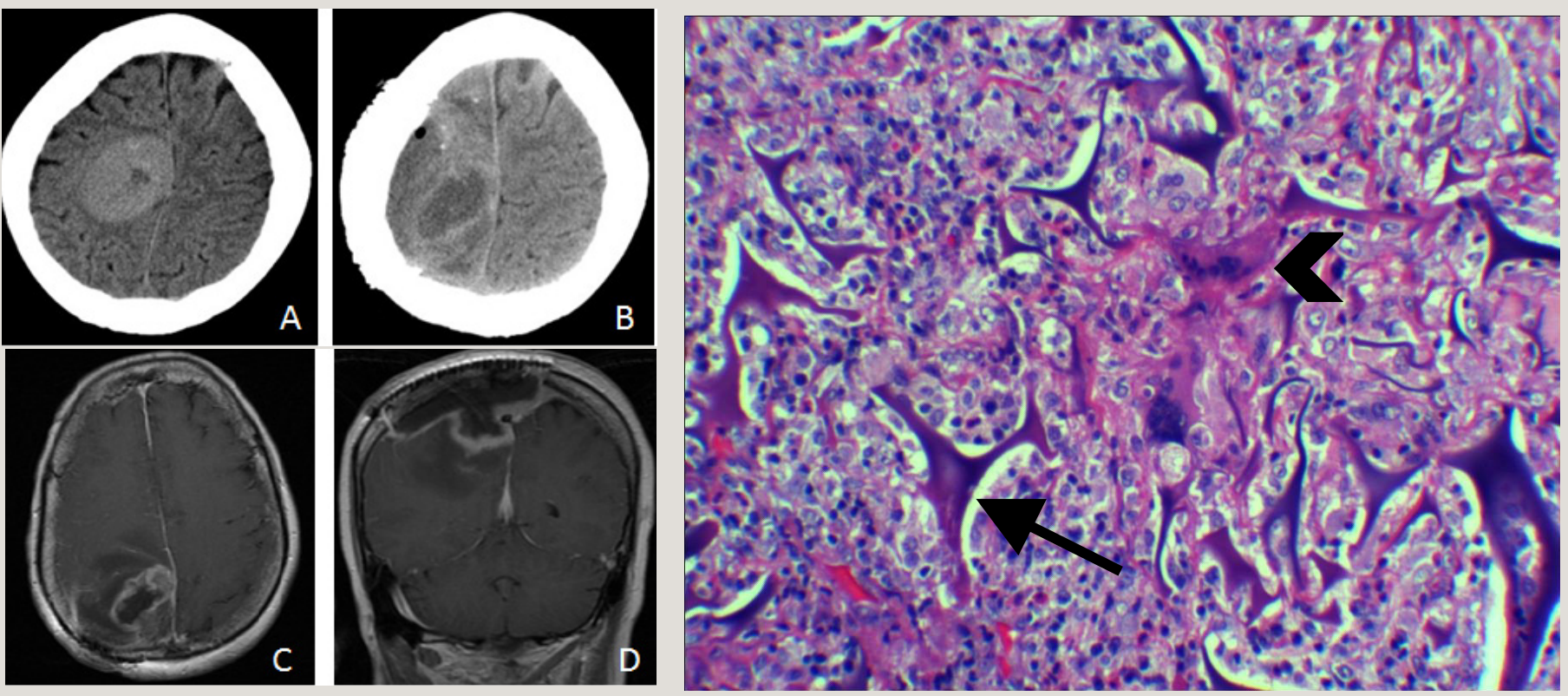

Figure 1. Axial CT preoperative right frontal meningioma (A), 5 weeks postoperative heterogeneous low attenuation mass (B). Post-contrast T1 - weighted MRI axial (C) and coronal (D) Figure 2. Resection specimen shows a granulomatous FB reaction. FB (arrow) and multinucleated giant cells (arrow head) were seen (HE x 200).

\section{CASE}

A 63-year-old woman underwent a craniotomy for a right frontal meningioma after developing left leg weakness and simple partial status epilepticus of the left leg (Figure 1A). Five weeks later, she developed recurrent status epilepticus on her left side. She de-

Corresponding author: Kessarin Panichpisal Contact Information: Kessarin@hotmail.com DOI: 10.12746/swrccc 2015.0310.132 nied fever and headache. Her examination revealed left hemiparesis. An unenhanced brain computed tomographic scan showed a discrete area of hypodensity with mild hyperdensity at its periphery and adjacent vasogenic edema and gas locules beneath the craniotomy site (Figure 1B). Gadolinium enhanced T1-weighted magnetic resonance images showed a peripherally enhancing lesion with vasogenic edema of the right centrum semiovale, possibly secondary to increased mass effect from extra-axial fluid collection at the operative site (Figure 1C-D). Additional 
surgery revealed extra-axial tissue with the macroscopic appearance of hemostatic agents and cerebral edema. Histopathological examination showed acute and chronic inflammation with extensive foreign body (FB) granulomas without residual tumor (Figure 2). The postoperative course was uneventful. The tissue culture was negative. Her symptoms improved significantly after surgery.

\section{Discussion}

Intracranial FB granulomas are very rare. Foreign bodies previously reported as causes of intracranial granulomas include hemostatic agents, dura mater substitutes, chemotherapy wafers, and agents employed for embolization of highly vascular tumors or arteriovenous malformations, such as polyvinyl alcohol, or sutures, or $N$-butyl-2-cyanoacrylate ( $N$-BCA Histoacryl, B. Braun, Melsungen, Germany). ${ }^{1,2} \mathrm{He}-$ mostatic agents are the most common causes of intracranial FB granulomas. Three most common resorbable hemostatic agents used are gelatin foam, oxidized cellulose, and microfibrillar collagen. ${ }^{1}$ In our case, the combination of gelatin foam (Gelfoam ${ }^{\circledR}$, Pfizer Inc., New York, NY), oxidized cellulose (Surgicel $($, Johnson and Johnson, Farmington, CT) and porcine gelatin flowable matrix (Surgiflo $\AA$, Johnson and Johnson, Farmington, CT) was used. These agents may induce an excessive inflammatory reaction in the vicinity of the surgical site, which potentially produces a clinically symptomatic and radiographically apparent lesion that could mimic recurrent tumor. ${ }^{1}$ Intracranial FB granulomas can present at any time from the intermediate postoperative period to several decades after initial surgery. ${ }^{1}$ The signal intensities of FB granulomas are variable on T1- and T2-weighted images reflecting a complex pathology, including acute or chronic inflammation, granuloma formation, fibrosis, collagen deposition, and degeneration of foreign materials. However, such granulomas invariably show nodular or ring-enhancing mass lesions. ${ }^{2}$ Hence FB granulomas should be included in the differential diagnosis of an enhancing mass arising after intracranial surgery, in addition to recurrent tumor, brain abscess, or radiation necrosis. ${ }^{1}$
Author affiliations : Kessarin Panichpisal is an interventional neurologist at the Asia Pacific Comprehensive Stroke Institute, Pomona, CA. Guy Aristide is in the Department of Internal Medicine, SUNY Downstate Medical Center, University Hospital of Brooklyn-Long Island College Hospital Brooklyn, NY. Craig Linden is in the Department of Radiology, SUNY Downstate Medical Center, Brooklyn, NY. John Miller is in the Division of Neurosurgery, Kingsbrook Jewish Medical Center \& Lutheran Medical Center, Brooklyn, NY.

Submitted: $1 / 6 / 2015$

Accepted: 2/1/2015

Reviewers: Eman Attaya MD

Conflict of Interest: None

Published electronically: 4/15/2015

\section{References}

1. Ribalta T, McCutcheon IE, Neto AG, et al. Textiloma (gossypiboma) mimicking recurrent intracranial tumor. Arch Pathol Lab Med 2004; 128:749-58.

2. Nannapaneni R, Satheesan K, Nath FP. Lintene granuloma following microvascular decompression mimicking a cerebellopontine angle tumour. J Clin Neurosci 2006; 13:380-3. 